\title{
Higgsophilic gauge bosons and monojets at the LHC
}

\author{
Jong Soo Kim, ${ }^{a}$ Oleg Lebedev ${ }^{b}$ and Daniel Schmeier ${ }^{c}$ \\ ${ }^{a}$ Instituto de Física Teórica UAM/CSIC, \\ Madrid, Spain \\ ${ }^{b}$ Department of Physics and Helsinki Institute of Physics, \\ Gustaf Hällströmin katu 2, FIN-00014 University of Helsinki, Finland \\ ${ }^{c}$ Physikalisches Institut and Bethe Center for Theoretical Physics, \\ University of Bonn, Bonn, Germany \\ E-mail: jong.kim@csic.es, oleg.lebedev@helsinki.fi, daschm@uni-bonn.de
}

ABSTRACT: We consider a generic framework where the Standard Model (SM) coexists with a hidden sector endowed with some additional gauge symmetry. When this symmetry is broken by a scalar field charged under the hidden gauge group, the corresponding scalar boson generally mixes with the SM Higgs boson. In addition, massive hidden gauge bosons emerge and via the mixing, the observed Higgs-like mass eigenstate is the only known particle that couples to these hidden gauge bosons directly. We study the LHC monojet signatures of this scenario and the corresponding constraints on the gauge coupling of the hidden gauge group as well as the mixing of the Higgs scalars.

KeYwords: Higgs Physics, Beyond Standard Model

ArXiv EPrint: 1507.08673 


\section{Contents}

1 Introduction 1

2 Models for Higgsophilic $Z^{\prime} \quad 2$

$2.1 Z^{\prime}$ from a hidden $\mathrm{U}(1)^{\prime} \quad 2$

2.2 Higgsophilic gauge bosons from $\mathrm{SU}(\mathrm{N})^{\prime} \quad 4$

$\begin{array}{lll}2.3 & \text { Perturbativity bounds } & 6\end{array}$

$\begin{array}{lll}3 & \text { LHC monojet constraints } & 6\end{array}$

$3.1 \operatorname{BR}\left(h_{2} \rightarrow\right.$ invisible $) \quad 7$

3.2 Current constraints from the LHC at $8 \mathrm{TeV} \quad 8$

3.3 Future limits from the $\mathrm{LHC}$ at $14 \mathrm{TeV} \quad 8$

4 Conclusion $\quad 12$

\section{Introduction}

When it comes to singlet scalar extentions of the Standard Model (SM), the SM Higgs field $H$ plays a special role: since $H^{\dagger} H$ is the only Lorentz and gauge invariant SM operator with mass dimension less than four, it is only the SM Higgs that can couple to extra SM singlet scalars $X$ at the renormalizable level [1-3]. This has interesting implications for the Higgs evolution in the early Universe, for example if the extra singlet is an inflaton [4].

Suppose that the extra singlet scalar $X$ is linked to a "hidden sector", which itself embeds a new gauge group $G_{N}$. If we are to probe the gauge bosons associated with this hidden gauge group, the feature described above can be used to construct a "Higgs Portal": here, $H$ is the only SM field that couples directly to these gauge bosons. Naïvely, one could give a hidden sector charge to $H$ to make it couple to the $G_{N}$ gauge bosons. However, in that case gauge invariance of the SM Yukawa couplings would require the SM fermions to be charged under $G_{N}$ as well. They would hence couple to the hidden gauge sector too and thus violate the assumption of a Higgs Portal.

A viable alternative would instead be to let $X$ be charged under $G_{N}$ and mix with the SM $H$. In fact, the Higgs portal coupling

$$
\Delta V=\lambda_{h x} H^{\dagger} H X^{\dagger} X
$$

is renormalizable, Lorentz invariant and complies with both the hidden sector and the SM gauge symmetries. As such it can and should be included in any theory describing the Standard Model and a hidden sector of the above type.

Assuming that both $H$ and $X$ develop vacuum expectation values (vevs), this coupling automatically leads to a mixing between the two fields upon spontaneous breaking of both 
symmetries. Therefore the observable mass eigenstates can couple to both the SM and the hidden sector. Most importantly, the recently observed SM-like Higgs boson can couple to the massive gauge bosons of the broken $G_{N}$, while all other SM particles cannot. We thus call the hidden sector gauge bosons "Higgsophilic". ${ }^{1}$ If they are themselves stable or only decay into other stable hidden sector fields, their production at colliders would appear as missing net momentum in the event. In this work, we study a particular LHC signature of the hidden sector using this argument, namely the production of a single jet associated with sizable missing transverse momentum, also called "monojet search".

Previous studies of related models include [6], where a kinetic mixing between the bosons associated to the SM U(1) and a hidden sector $\mathrm{U}(1)^{\prime}$ boson was assumed. In that case however, the SM Higgs boson is not the only SM field that couples to the hidden sector which entails different collider signatures. LHC studies of a specific limit in which the second Higgs scalar decouples [7] was considered in [8], in which case the obtained estimates are considerably optimistic (see also $[9,10]$ ). A recent paper [11] presents an interesting related LHC study focusing on the vector boson fusion (VBF) channel in a specific dark matter (DM) scenario and a different kinematic range than discussed here.

In our work, we consider a more general framework in which the heavy Higgs-scalar couples to arbitrary gauge bosons of the hidden sector and we improve on previous studies in technical aspects. After introducing the model and its phenomenological implications in section 2 we discuss the methodology and the results of our collider study in section 3 .

\section{Models for Higgsophilic $Z^{\prime}$}

\section{$2.1 \quad Z^{\prime}$ from a hidden $\mathrm{U}(1)^{\prime}$}

Lagrangian, mass eigenstates and couplings: we outline the most important phenomenological results for our model below. For more details, see e.g. [12].

Consider the Standard Model extended by a hidden sector with a sequestered $\mathrm{U}(1)^{\prime}$, which by construction is orthogonal to the SM U(1). The hidden sector contains the vector gauge field $A_{\mu}^{\prime}$ associated with the $\mathrm{U}(1)^{\prime}$ and a complex scalar $X$ charged under the $\mathrm{U}(1)^{\prime}$ but neutral under the SM gauge group. The kinetic terms of these fields read

$$
\mathcal{L}_{\text {kin }}=-\frac{1}{4} F_{\mu \nu}^{\prime} F^{\prime, \mu \nu}+\left(D_{\mu} X\right)^{\dagger} D^{\mu} X,
$$

with the covariant derivative $D_{\mu} X \equiv\left(\partial_{\mu}-i \tilde{g} A_{\mu}^{\prime}\right) X$ and $\tilde{g}$ being the gauge coupling associated with the hidden gauge group.

The hidden scalar $X$ and the SM Higgs field $H$ have a common scalar potential. In unitary gauge we define $H^{T}=(0, h / \sqrt{2}), X=x / \sqrt{2}$ with real scalar fields $h$ and $x$. The full scalar potential containing all terms of mass dimension 4 that are consistent with the symmetries of the model reads

$$
V=\frac{1}{4} \lambda_{h} h^{4}+\frac{1}{4} \lambda_{h x} x^{2} h^{2}+\frac{1}{4} \lambda_{x} x^{4}+\frac{1}{2} m_{h}^{2} h^{2}+\frac{1}{2} m_{x}^{2} x^{2} .
$$

\footnotetext{
${ }^{1}$ The term "Higgsophilic" has first appeared in [5] in a different context.
} 
Here the real parameters $\lambda_{i}$ and $m_{i}^{2}$ are the quartic couplings and mass terms, respectively. The scalar potential is such that not only $H$ but also $X$ develops a vev:

$$
\langle h\rangle=v,\langle x\rangle=u .
$$

Upon spontaneous breaking of $\mathrm{U}(1)^{\prime}$ via $X$, the corresponding Goldstone boson is absorbed by the $\mathrm{U}(1)^{\prime}$ gauge field, leaving us with one real scalar degree of freedom and a massive vector field, which we will call $Z_{\mu}^{\prime}$ from now on.

Using the minimisation conditions of the scalar potential and expanding both fields around their vevs, one finds the mass matrix of the scalar sector whose eigenvalues are given by

$$
m_{1,2}^{2}=\lambda_{h} v^{2}+\lambda_{x} u^{2} \mp \sqrt{\left(\lambda_{x} u^{2}-\lambda_{h} v^{2}\right)^{2}+\lambda_{h x}^{2} u^{2} v^{2}} .
$$

Defining the mass eigenstates by the following rotation

$$
\begin{aligned}
& h_{1}=h \cos \theta+x \sin \theta, \\
& h_{2}=h \sin \theta-x \cos \theta,
\end{aligned}
$$

the mixing angle $\theta$ is given by

$$
\tan 2 \theta=\frac{\lambda_{h x} u v}{\lambda_{h} v^{2}-\lambda_{x} u^{2}}
$$

The definition of $\theta$ is such that in the limit $\theta=0$ the lighter of the two eigenstates is the SM-like Higgs boson. ${ }^{2}$

Setting the $\mathrm{U}(1)^{\prime}$ charge of $X$ to +1 we find

$$
m_{Z^{\prime}}=\tilde{g} u
$$

and the following trilinear $S V V$ interactions relevant for our study

$$
\Delta \mathcal{L}=\tilde{g} m_{Z^{\prime}} \sin \theta h_{1} Z_{\mu}^{\prime} Z^{\prime \mu}-\tilde{g} m_{Z^{\prime}} \cos \theta h_{2} Z_{\mu}^{\prime} Z^{\prime \mu} .
$$

These vertices are responsible for production of pairs of $Z^{\prime}$ s at the LHC.

Signatures of a $\boldsymbol{h} \boldsymbol{Z}^{\prime} \boldsymbol{Z}^{\prime}$ coupling: an important constraint on the model comes from the branching ratio of the invisible Higgs decays [9] which only applies if $m_{Z^{\prime}}<m_{1} / 2$, that is if the hidden sector gauge boson is lighter than about $63 \mathrm{GeV}$. Bounds from the "standard" $Z^{\prime}$ searches do not apply to the Higgsophilic case as they assume couplings of the $Z^{\prime}$ to SM fermions and/or SM gauge bosons [13].

As outlined in the introduction, if the $Z^{\prime}$ is stable or decays into hidden sector states, the corresponding signature at a hadron collider would be missing transverse momentum which can, for instance, be observed in conjunction with a jet. The main purpose of this paper is to study the LHC sensitivity to this final state.

\footnotetext{
${ }^{2}$ Note that this convention for $\theta$ differs from the one used in [12].
} 
Dark matter candidates: in our framework, there exist several candidates for dark matter. A straightforward possibility is that the massive gauge fields themselves constitute DM. In the Abelian case, only pairs of $Z^{\prime}$ couple to $h_{1}, h_{2}$, i.e. there exists a $\mathbb{Z}_{2}$-parity [7] which can be traced back to charge conjugation symmetry:

$$
Z_{\mu}^{\prime} \rightarrow-Z_{\mu}^{\prime}
$$

This renders the $Z^{\prime}$ stable and weakly coupled to the Standard Model, which are the prerequisites of viable DM candidates. The heavy $h_{2}$ limit of this set-up was studied in [7], where it was found that all of the DM constraints can be satisfied for sub-TeV $Z^{\prime}$ masses (see also $[14,15])$. A recent analysis of the $\mathrm{U}(1)^{\prime}$ case can be found in $[16,17]$.

Another approach to the DM problem is to consider additional fields in the hidden sector that can account for DM. For instance, "hidden fermions" $\chi$ charged under U(1)' can couple to the Higgsophilic gauge fields as follows

$$
\Delta \mathcal{L}=\tilde{g} \bar{\chi} \gamma^{\mu} A_{\mu}^{\prime} \chi
$$

In that case, after spontaneous symmetry breaking the massive $Z^{\prime}$ can decay into fermionic DM $\chi$. In terms of collider phenomenology, this leads to the same missing $E_{T}$ signatures and hence would not change the results of this study. There are however differences in direct DM detection as the hidden fermions have loop-suppressed interactions with nucleons compared to those of the $Z^{\prime}$.

DM constraints on our model depend on additional assumptions such as the nature of dark matter and its production mechanism(s) in the Early Universe. In this work, we set these issues aside and focus exclusively on the collider aspects of our framework.

\subsection{Higgsophilic gauge bosons from $\mathrm{SU}(\mathrm{N})^{\prime}$}

Abelian case parallel: the above considerations can straightforwardly be generalized to the non-Abelian case: suppose we have an $\mathrm{SU}(\mathrm{N})^{\prime}$ symmetry in the hidden sector instead of the $\mathrm{U}(1)^{\prime}$. We now take $X$ to be an N-plet transforming in the fundamental representation of SU(N) ${ }^{\prime}$. The covariant derivative in eq. (2.1) then changes to $D_{\mu}=\partial_{\mu}-i \tilde{g} A_{\mu}^{a} T^{a}$, where $A_{\mu}^{a}$ are the $N^{2}-1$ vector fields and $T^{a}$ are the group generators satisfying ${ }^{3} \operatorname{Tr}\left(T^{a} T^{b}\right)=2 \delta^{a b}$.

A vev of $X$ breaks $\mathrm{SU}(\mathrm{N})^{\prime} \rightarrow \mathrm{SU}(\mathrm{N}-1)^{\prime}$. In unitary gauge, $X$ is expressed as

$$
X=\frac{1}{\sqrt{2}}\left(\begin{array}{c}
0 \\
\vdots \\
0 \\
x
\end{array}\right)
$$

with $x$ being a real scalar field which gets a vev, analogously to the $\mathrm{U}(1)^{\prime}$ case. From the pattern of symmetry breaking, it is clear that $2 N-1$ degrees of freedom of $X$ get absorbed and lead to $2 N-1$ massive gauge fields, while the remaining degree of freedom corresponds to the "hidden sector Higgs" boson.

\footnotetext{
${ }^{3}$ We use this normalization for easier translation from the Abelian case with charge +1 . This differs from the normalization used in [16] which is obtained by the replacement $\tilde{g}^{2} \rightarrow \tilde{g}^{2} / 4$.
} 
In this gauge, the scalar potential is identical to that for the Abelian case (2.2) and thus the conclusions that follow from that equation also apply. The only difference is that now $h_{1}$ and $h_{2}$ couple to $2 N-1$ mass degenerate bosons such that eq. (2.8) now reads

$$
\Delta \mathcal{L}=\sum_{i=1}^{2 N-1}\left(\tilde{g} m_{Z^{\prime}} \sin \theta h_{1} Z_{i, \mu}^{\prime} Z_{i}^{\prime \mu}-\tilde{g} m_{Z^{\prime}} \cos \theta h_{2} Z_{i, \mu}^{\prime} Z_{i}^{\prime \mu}\right) .
$$

Since $Z_{i, \mu}^{\prime}$ are indistinguishable experimentally, this effectively amounts to replacing

$$
\tilde{g}^{2} \rightarrow(2 N-1) \tilde{g}^{2}
$$

in cross sections and decay width calculations of the Abelian case. This expression bears resemblance to the 't Hooft coupling [18] $\lambda=\tilde{g}^{2} N$ for large $N$.

Residual SU $(\mathbf{N}-\mathbf{1})^{\prime}$ : at this stage, the other $(N-1)^{2}-1$ gauge bosons remain massless. As they have no coupling to $h_{1,2}$ they do not play any role in our collider analysis. However, they would affect the cosmological history of our Universe and thus necessitate further discussion.

In order to break the gauge group completely, one may invoke not just 1 but $N-1$ hidden sector Higgs fields $X_{k}$ in the fundamental representation of $\mathrm{SU}(\mathrm{N})^{\prime}$. When all of them get vevs, the symmetry gets broken completely and all vector fields acquire mass. In general, all the remaining scalar degrees of freedom mix independently with the Higgs, leading to a highly entangled scalar sector. However, one would not expect all the mixings to be equally important. Hence, it is reasonable to make the simplifying assumption that the mixing is dominated by one $N$-plet which we choose to be the one in eq. (2.11). In this case, one may still neglect the production of the remaining $(N-1)^{2}-1$ gauge bosons at the LHC and the above result holds.

Alternatively one could assume condensation of $\mathrm{SU}(\mathrm{N}-1)^{\prime}$ at low energies which would make the relevant degrees of freedom massive (similarly to "glueballs" in QCD).

Either way we may focus on the couplings of $h_{1}$ and $h_{2}$ to $2 N-1$ massive vector bosons and ignore the rest. The only difference from the Abelian case would be the replacement in eq. (2.13).

Dark matter candidates: consider for example $N=2$ : as shown in [19], our considerations of the Abelian gauge field DM equally apply to $\mathrm{SU}(2)$ as long as the symmetry is broken by a single $\mathrm{SU}(2)$ doublet. In this case, the gauge fields couple to the physical scalars in pairs which renders the $Z_{i}^{\prime}$ stable.

Although the triple gauge vertex breaks an analog of the $\mathbb{Z}_{2}$-parity in eq. (2.9), the interactions preserve a related $\mathbb{Z}_{2} \times \mathbb{Z}_{2}$ symmetry,

$$
\begin{aligned}
& A_{\mu}^{1} \rightarrow-A_{\mu}^{1}, \quad A_{\mu}^{2} \rightarrow-A_{\mu}^{2}, \\
& A_{\mu}^{1} \rightarrow-A_{\mu}^{1}, \quad A_{\mu}^{3} \rightarrow-A_{\mu}^{3},
\end{aligned}
$$

where the upper index refers to the $\mathrm{SU}(2)$ adjoint generators. This symmetry is sufficient to ensure stability of DM, while it actually generalizes to a custodial $\mathrm{SO}(3)$ [19]. Phenomenology of the SU(2) DM was studied in [19], see also [20]. 
The general $\mathrm{SU}(\mathrm{N})^{\prime}$ case was analyzed in [16]. It was found that the symmetries that stabilize DM include both inner and outer automorphisms of $\mathrm{SU}(\mathrm{N})^{\prime}$. These remain valid symmetries of the theory if $\mathrm{CP}$ is unbroken in the hidden sector. The resulting stable gauge fields are again viable DM candidates [16].

As in the Abelian case, there is the option of having additional hidden sector fields $\chi$ charged under the $\mathrm{SU}(\mathrm{N}-1)^{\prime}$ which could constitute dark matter.

\subsection{Perturbativity bounds}

We conclude this section with a few words on the relevant theory limits. Requiring the hidden sector to be perturbative at the LHC energies implies a bound on the 't Hooft coupling,

$$
\tilde{g}^{2} N<4 \pi^{2}
$$

where $4 \pi^{2}$ represents the loop factor appearing at each order in perturbation theory. The Abelian case emerges trivially from eq. (2.15) by setting $N=1$.

Further perturbativity constraints should be imposed on the scalar quartic couplings in eq. (2.2). These however are relevant only if there is a significant hierarchy between the gauge boson mass and $m_{2}[16]$ which we are not going to consider here.

To conclude this section, let us summarize the new features of our set-up. Although certain collider aspects of the $\mathrm{U}(1)$ hidden sector have been studied before (see e.g. [8]), an analysis of the $\mathrm{SU}(\mathrm{N})$ case is missing. We find that for "hidden Higgs" fields in the fundamental representation, the $\mathrm{U}(1)$ results also apply to the $\mathrm{SU}(\mathrm{N})$ case up to the rescaling of the gauge coupling. Furthermore, our analysis holds for a more general situation of decaying gauge fields as long as the decay products do not couple to the SM fields.

\section{LHC monojet constraints}

Constraints on the model depend strongly on the Higgsophilic gauge boson mass. If it is lighter than about $63 \mathrm{GeV}$, the SM-like scalar $h_{1}$ can decay into pairs of $Z^{\prime}$ s. In that case, experimental constraints are rather strong and can be extracted from the results of [9]. For example, for $m_{Z^{\prime}} \sim 50 \mathrm{GeV}, \tilde{g} \sin \theta$ can be at most of order $10^{-2}$. A more recent analysis of this decay mode can be found in [11].

The mixing angle $\theta$ is constrained by the current Higgs coupling measurements, $\sin \theta<$ 0.44 [21]. The analogous bound from the heavy Higgs searches is sensitive to the invisible $h_{2}$ decay branching fraction which results in a weaker constraint. Further bounds on the model parameters can be derived if one makes assumptions about the nature of dark matter. In particular, if the $Z^{\prime}$ constitutes all of dark matter, the direct detection experiments require $\tilde{g} \lesssim \mathcal{O}\left(10^{-1}\right)$ for $m_{Z^{\prime}} \sim 100 \mathrm{GeV}$ and $\sin \theta \sim 0.3$ [16]. However, this bound is lifted if the gauge bosons contribute only a fraction of the total dark matter density or if DM is composed of other hidden sector fields, as discussed in section 2.1.

In what follows, we focus on the regime

$$
m_{1}<2 m_{Z^{\prime}}<m_{2}
$$




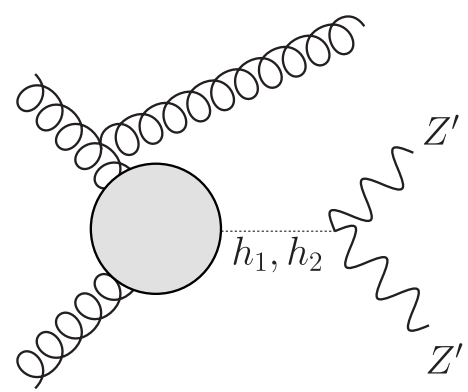

Figure 1. Main contribution to the monojet production with missing $E_{T}$.

in which case the decay $h_{2} \rightarrow Z^{\prime} Z^{\prime}$ is allowed and its width is enhanced by powers of $m_{2} / m_{Z^{\prime}}$ characteristic of the pseudo-Goldstone boson production. For heavier $Z^{\prime}$, the production cross section is too small to have interesting constraints (see also [11]). Among other things, $Z^{\prime}$ production via off-shell Higgses suffers from destructive interference between the $h_{1}$ and $h_{2}$ contributions (see also [22]).

Here, we focus on the monojet signature shown in figure 1. Other channels, such as Higgs production through vector boson fusion and $h_{2}$ visible decays, can provide further important information about the model but are not discussed in this work. Related studies of fermion DM production have recently appeared in [23].

We consider $Z^{\prime}$ pair production in association with one hard jet via on-shell heavy Higgs production,

$$
p p \rightarrow h_{2} j \rightarrow Z^{\prime} Z^{\prime} j,
$$

where $j$ denotes a parton level jet. The $Z^{\prime}$ will not be detected at the LHC and thus the corresponding signal is one large transverse momentum jet and large transverse missing momentum which is back to back to the jet. Note that additional jets can arise from strong initial state radiation and thus the above signal can be accompanied by further softer jets.

In the following, we first consider the invisible decay branching ratio for $h_{2}$. Then we discuss constraints from the current monojet searches with $8 \mathrm{TeV}$ LHC data and the prospects at $14 \mathrm{TeV}$ assuming an integrated luminosity of $600 \mathrm{fb}^{-1}$.

\section{1 $\operatorname{BR}\left(h_{2} \rightarrow\right.$ invisible $)$}

When allowed kinematically, $h_{2}$ decays into SM particles, pairs of $Z^{\prime}$ as well as pairs of $h_{1}$. Details of the relevant couplings and decay rate formulae for $h_{2} \rightarrow h_{1} h_{1}$ can be found in [21].

The coupling between the light and heavy Higgses is given by

$$
\Delta V=\frac{2 m_{1}^{2}+m_{2}^{2}}{2 v} \sin \theta\left(\cos ^{2} \theta+\frac{v}{u} \sin \theta \cos \theta\right) h_{1}^{2} h_{2}
$$

Here $v$ is the SM Higgs vev and $u$ is determined by the Higgsophilic gauge boson mass according to eq. (2.7). The corresponding $h_{2} \rightarrow h_{1} h_{1}$ decay rate is then

$$
\Gamma\left(h_{2} \rightarrow h_{1} h_{1}\right)=\frac{\left(2 m_{1}^{2}+m_{2}^{2}\right)^{2}}{32 \pi v^{2} m_{2}^{2}} \sin ^{2} \theta\left(\cos ^{2} \theta+\frac{v}{u} \sin \theta \cos \theta\right)^{2} \sqrt{1-\frac{4 m_{1}^{2}}{m_{2}^{2}}} .
$$


From eq. (2.8) we find the decay width for $h_{2} \rightarrow Z^{\prime} Z^{\prime}$ to be

$$
\Gamma\left(h_{2} \rightarrow Z^{\prime} Z^{\prime}\right)=\frac{\tilde{g}^{2} \cos ^{2} \theta m_{2}^{3}}{32 \pi m_{Z^{\prime}}^{2}} \sqrt{1-\frac{4 m_{Z^{\prime}}^{2}}{m_{2}^{2}}}\left(1-\frac{4 m_{Z^{\prime}}^{2}}{m_{2}^{2}}+\frac{12 m_{Z^{\prime}}^{4}}{m_{2}^{4}}\right) .
$$

Finally, the width of the $h_{2}$ decay into SM particles is obtained by rescaling the heavy SM Higgs result,

$$
\Gamma\left(h_{2} \rightarrow \mathrm{SM}\right)=\sin ^{2} \theta \Gamma_{\mathrm{SM}}\left(m_{h}=m_{2}\right) .
$$

Eqs. (3.4)-(3.6) determine the invisible decay branching ratio for $h_{2}$ :

$$
\mathrm{BR}\left(h_{2} \rightarrow \text { invisible }\right)=\left(1+\frac{\Gamma\left(h_{2} \rightarrow h_{1} h_{1}\right)+\Gamma\left(h_{2} \rightarrow \mathrm{SM}\right)}{\Gamma\left(h_{2} \rightarrow Z^{\prime} Z^{\prime}\right)}\right)^{-1} .
$$

For small $\sin \theta$, the SM channels as well as $h_{2} \rightarrow h_{1} h_{1}$ are suppressed by $\sin ^{2} \theta$ and the $h_{2} \rightarrow Z^{\prime} Z^{\prime}$ mode typically dominates. In addition, the decay of $h_{2}$ into vector particles is enhanced by the usual $m_{2} / m_{Z^{\prime}}$ factor associated with the would-be Goldstone boson production.

To give an example, for $m_{2}=300 \mathrm{GeV}$, the SM heavy Higgs width is $\Gamma_{\mathrm{SM}} \simeq 10 \mathrm{GeV}$. Then for $\tilde{g}=1, m_{Z^{\prime}}=100 \mathrm{GeV}$ and $\sin \theta=0.4$, the $h_{2}$ invisible decay branching ratio exceeds $90 \%$.

\subsection{Current constraints from the LHC at $8 \mathrm{TeV}$}

The ATLAS and CMS collaborations have presented limits on invisible Higgs decays using $20.3 \mathrm{fb}^{-1}$ of data at $\sqrt{s}=8 \mathrm{TeV}$ [24-28]. No excess above the SM has been observed, and CMS and ATLAS have derived 95\% C.L. limits on the production cross section times the branching ratio as a function of the Higgs-like boson mass. In particular, figure 16 of [24] shows that the SM Higgs boson with the hypothetical invisible branching ratio of $100 \%$ is allowed by the monojet data. In our scenario, the production mechanism is the same as that in the SM. However, the additional factor of the mixing between the SM-like Higgs and the singlet heavily suppresses the production rate and thus no limits on our model can be derived from $8 \mathrm{TeV}$ data.

\subsection{Future limits from the $\mathrm{LHC}$ at $14 \mathrm{TeV}$}

In this subsection, we discuss prospects of constraining our Higgs portal model at the LHC with $\sqrt{s}=14 \mathrm{TeV}$. We extrapolate an existing ATLAS monojet search at $8 \mathrm{TeV}$ to $14 \mathrm{TeV}$ adding signal regions with stricter cuts on the transverse missing energy and the leading jet but without optimizing the selection cuts.

Simulation: while the more recent monojet study [24] puts limits on an invisibly decaying SM-like Higgs boson, for our $14 \mathrm{TeV}$ projection we have closely followed the slightly older monojet search of ref. [29]. By the time ref. [24] was published the SM backgrounds for this study had already been fully simulated based on ref. [29]. The simulation of SM backgrounds with large cross sections requires large computer resources and since both 


\begin{tabular}{|l|c|c|c|c|c|}
\hline Cut & M1 & M2 & M3 & M4 & M5 \\
\hline lepton veto & \multicolumn{5}{|c|}{ yes } \\
$N_{j}\left(p_{T}>30 \mathrm{GeV},|\eta|<2.8\right)$ & \multicolumn{5}{|c|}{$\leq 3$} \\
$\Delta \phi\left(\vec{p}_{\text {jet }}, \vec{p}_{T}^{\text {miss }}\right)$ & \multicolumn{5}{|c|}{$>0.4$} \\
\hline$p_{T}($ leading jet $)$ in $\mathrm{GeV}$ & $\geq 280$ & $\geq 320$ & $\geq 450$ & $\geq 500$ & $\geq 550$ \\
$E_{T}^{\text {miss }}$ in $\mathrm{GeV}$ & $\geq 220$ & $\geq 320$ & $\geq 450$ & $\geq 500$ & $\geq 550$ \\
\hline
\end{tabular}

Table 1. Selection cuts used for the $\sqrt{s}=14 \mathrm{TeV}$ monojet analysis.

studies have similar selection cuts (apart from the details of the jet veto) we have decided to adhere to ref. [29] in this work.

We have implemented the relevant kinematic selection cuts for the signal regions in this study. All monojet signal regions demand a lepton veto and a maximum of three jets with $p_{T}>30 \mathrm{GeV}$. An additional requirement is imposed on the azimuthal angle between the missing transverse vector and the jets, $\Delta \phi\left(\right.$ jet, $\left.\mathrm{p}_{\mathrm{T}}^{\mathrm{miss}}\right)>0.4$, in order to suppress the QCD multijet background. Finally, five signal regions M1, M2, M3, M4 and M5 are defined with increasing cuts on the transverse momentum of the leading jet and the total missing transverse energy of the event. They are listed in table 1.

We have generated the parton level signal events within the POWHEG2 framework [3032] which then have been passed to Pythia6.4 [33]. We have produced $g g \rightarrow h_{2}$ [34] and $V V \rightarrow h_{2}[35]$ samples. Since the $V h_{2}$ production mechanism is subdominant, we have omitted the production channel of $h_{2}$ in association with a gauge boson. The $g g \rightarrow h_{2}$ sample dominates the total production cross section. The cross sections for the various signal production modes have been taken from [36]. The signal event generation has been validated against the results on invisible Higgs decays from [24] before generating signal events for the $14 \mathrm{TeV}$ study.

Let us briefly discuss the major SM backgrounds for the $14 \mathrm{TeV}$ study. The main background is the $Z$ boson production in association with one jet where the $Z$ decays into a pair of neutrinos. The $W j$ production with the $W$ decaying leptonically also contributes significantly, most importantly via the decay into a tau and a neutrino. $t \bar{t}$ events give a small contribution but are important for choosing the cuts for the signal regions. We omit the single top background since its cross section is by a factor of 4 smaller than the $t \bar{t}$ background which itself only contributes at the percent level. For the same reason, we have neglected the $Z / \gamma^{*} j$ and SM diboson as well as dijet/trijet QCD backgrounds.

We estimate the dominant SM backgrounds as follows: the $W j$ and $Z j$ backgrounds are generated with Sherpa2.1.1 [37] including up to 3 partons with CTEQ10 PDF [38]. The $t \bar{t}$ background has been simulated with POWHEG2 [39] and the parton level events were passed to Pythia6.4.25 [33] with CTEQ6L1 parton distribution function [40]. The $t \bar{t}$ cross section has been determined with Top++2.0 [41].

Our $14 \mathrm{TeV}$ monojet analysis has been implemented into the CheckMATE1.2.1 framework [42]. CheckMATE uses the fast detector simulation Delphes3.10 [43] with heavily 


\begin{tabular}{|r|r|r|r|r|r|r|}
\hline SR & $Z j$ & $W j$ & $t \bar{t}$ & total & signal & $S / \sqrt{B}$ \\
\hline M1 & 2378934 & 2024466 & 67821 & 4471221 & 13268 & 6.3 \\
M2 & 742710 & 442296 & 13327 & 1198333 & 4894 & 4.5 \\
M3 & 207804 & 102852 & 2656 & 313312 & 1514 & 2.7 \\
M4 & 80730 & 30036 & 1118 & 111884 & 942 & 2.8 \\
M5 & 33252 & 11610 & 625 & 45487 & 594 & 2.8 \\
\hline
\end{tabular}

Table 2. Number of background and example signal events $\left(m_{2}=200 \mathrm{GeV}, \sin \theta=0.4\right.$ and $\mathrm{BR}\left(h_{2} \rightarrow\right.$ invisible $\left.)=1\right)$ in the signal regions M1 to M5 at the LHC with $\sqrt{s}=14 \mathrm{TeV}$ and an integrated luminosity of $600 \mathrm{fb}^{-1}$. In the last column, we have estimated the statistical significance with $S / \sqrt{B}$.

modified detector tunings of the ATLAS detector. For a given event sample, it determines the number of expected signal events passing the selection requirements. Its AnalysisManager feature allows for an easy implementation of new studies [44]. We have used AnalysisManager to implement the aforementioned selection cuts and obtain the expected background numbers. The latter is used by CheckMATE to automatically calculate the $\mathrm{CL}_{\mathrm{S}}$ value [45] in order to quantify the compatibility of the signal prediction with the observation, which for our prospective study equals the SM expectation. The statistical errors of the signal and of the background are taken into account. The uncertainty of the signal rate is controlled by the renormalization and factorization scale dependence of the signal cross section, parton distribution, jet and missing transverse energy scale as well as parton shower scale uncertainties. For current LHC studies (see e.g. [24]), this results in a total signal rate uncertainty of roughly $15 \%$. However, we believe that some of the theory predictions will become more precise in the long run and hence we assume a $10 \%$ theory error on the signal. The overall magnitude of the systematic errors and its relative contribution to the statistical uncertainty is hard to estimate for a future high luminosity LHC run. We therefore determine optimistic limits for negligible systematic errors and discuss their impact on the final result at the end of the section.

Benchmark parameters: to allow for a large phase space and a large coupling, we set $m_{Z^{\prime}}=65 \mathrm{GeV}$ in our benchmak study. Eq. (3.7) implies that the result depends on the combination $\tilde{g} / m_{Z^{\prime}}$ as long as $m_{2}^{2} \gg m_{Z^{\prime}}^{2}$, therefore our bounds for other values of $m_{Z^{\prime}}$ can be obtained by an appropriate rescaling of $\tilde{g}$. For heavier $Z^{\prime}$, the kinematic suppression factor must also be taken into account.

Obviously, the production cross section is sensitive to $\sin \theta$, for which we take two representative values, $\sin \theta=0.3,0.4$. The LHC constraints on $\sin \theta$ from invisible decays of a heavy Higgs depend on $m_{2}$ as well as $\mathrm{BR}\left(h_{2} \rightarrow\right.$ invisible $)$ since these searches are based on visible final states, e.g. photons and leptons. For large $\operatorname{BR}\left(h_{2} \rightarrow\right.$ invisible $)$, the usual bounds [21] relax and the above values for $\sin \theta$ are consistent with the data. 


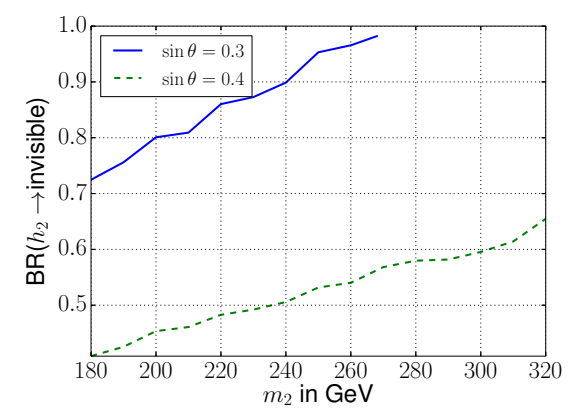

(a)

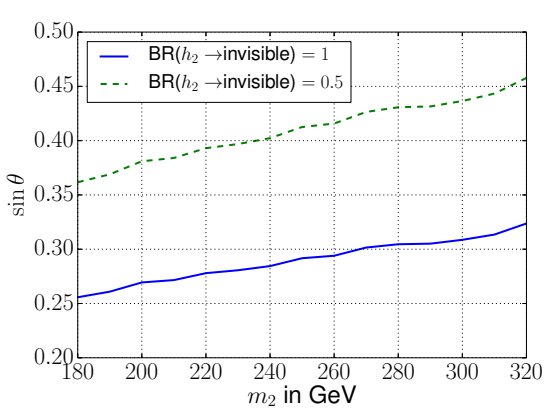

(b)

Figure 2. Expected future 95\% C.L. limits on the heavy Higgs boson at $\sqrt{s}=14 \mathrm{TeV}$ with an integrated luminosity of $600 \mathrm{fb}^{-1}$. a) Upper limits on $\mathrm{BR}\left(h_{2} \rightarrow\right.$ invisible) for fixed mixing angles. b) Upper limits on the mixing angle for fixed $h_{2}$ invisible decay branching ratios.

Results: in table 2, we list the total number of $Z+j, W+j, t \bar{t}$ and the sum of the background events for a signal benchmark point $m_{2}=200 \mathrm{GeV}, \sin \theta=0.4$ and $\mathrm{BR}\left(h_{2} \rightarrow\right.$ invisible $)=1$ at the LHC with $\sqrt{s}=14 \mathrm{TeV}$ for an integrated luminosity of $600 \mathrm{fb}^{-1}$. In our numerical studies, we determine the $95 \% \mathrm{CL}_{\mathrm{S}}$ limits. However, for illustration, in the last column we show the statistical significance of the signal estimated with $S / \sqrt{B}$, where $S$ and $B$ are the number of signal and background events, respectively. The $Z j$ and $W j$ production are the dominant SM backgrounds as expected. The $t \bar{t}$ background is heavily reduced by the jet veto [46] which makes it negligible. We obtain the best statistical significance, namely above six, in the signal region M1. One should keep in mind however that no systematic error has been included.

In figure 2(a), we present our 95\% C.L exclusion limits for $\mathrm{BR}\left(h_{2} \rightarrow\right.$ invisible) as a function of the $h_{2}$ mass for an integrated luminosity of $600 \mathrm{fb}^{-1}$. In this mass range, the LHC would be sensitive to invisible decay branching ratios down to $40 \%$ for $\sin \theta=0.4$. Heavier $h_{2}$ weaken the limits and lead to the sensitivity range determined by the point at which $\mathrm{BR}_{\text {limit }}=1$. As can be seen in figure $2(\mathrm{a})$, at $\sin \theta=0.3$ the monojet signal can be useful for $m_{2}$ up to $270 \mathrm{GeV}$.

The monojet bounds could also be interpreted from a different angle. One may assume that the hidden gauge coupling is large such that $\mathrm{BR}\left(h_{2} \rightarrow\right.$ invisible $) \simeq 1$. In this case, one would instead get a bound on $\sin \theta$ as shown in figure 2(b). Clearly, there are also other probes of $\sin \theta$ such as the $h_{1}$ couplings to matter which will likely set a stronger bound. When $\sin \theta$ is determined and the $h_{2}$ resonance is found, the interpretation of the monojet signal in terms of $\tilde{g} / m_{Z^{\prime}}$ becomes unambiguous.

The limits on the branching ratio are translated into the upper bounds on the coupling constant $\tilde{g}$ in figure $3(\mathrm{a})$, for the two chosen values of $\sin \theta$. Interestingly, $\tilde{g}$ as small as $10^{-1}$ could in principle be constrained. If, on the other hand, one assumes a large $\tilde{g}, \operatorname{BR}\left(h_{2} \rightarrow\right.$ invisible) probes a wide range of $m_{Z^{\prime}}$, as shown in figure 3(b), which can cover the entire kinematic reach $m_{Z^{\prime}} \leq m_{2} / 2$.

As explained in previous sections, the above limits can be reinterpreted in the nonAbelian case by taking into account the multiplicity factor in eq. (2.13). 


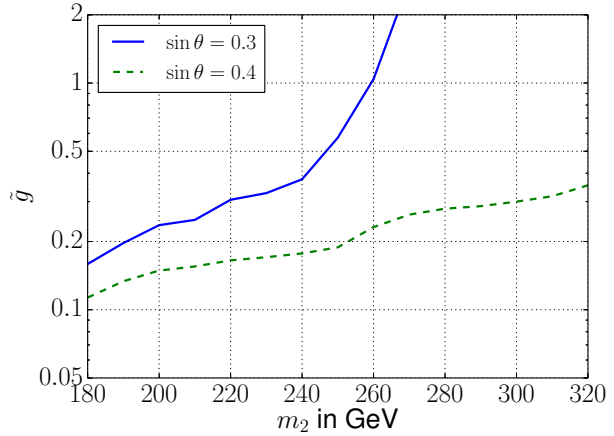

(a)

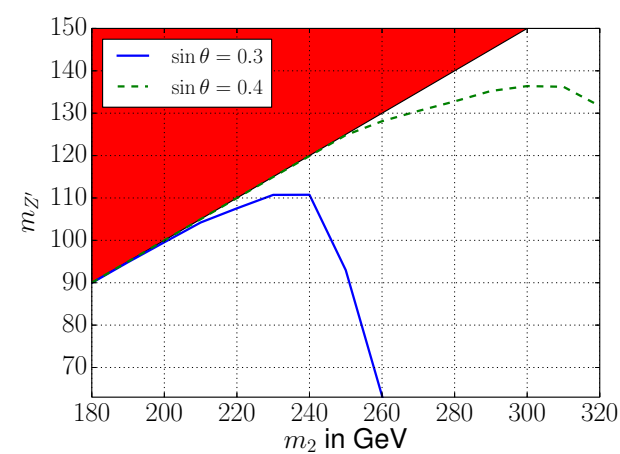

(b)

Figure 3. $95 \%$ C.L. future limits on the hidden gauge sector parameters at $\sqrt{s}=14 \mathrm{TeV}$ with an integrated luminosity of $600 \mathrm{fb}^{-1}$ and two representative values of $\sin \theta$. a) Upper limit on the hidden sector gauge coupling $\tilde{g}$ for $M_{Z^{\prime}}=65 \mathrm{GeV}$. b) Lower Limit on the hidden sector gauge boson mass for $\tilde{g}=1$. The red shaded area does not allow for a decay into two on-shell $Z^{\prime}$ bosons.

In our study, the signal to background ratio is roughly $S / B \lesssim 1 \%$. The shape of the signal and the dominant irreducible background process $Z(\rightarrow \nu \bar{\nu})+j$ as well as $W(\rightarrow$ $\tau \bar{\nu})+j$ are very similar which makes the signal extraction extremely difficult. In the above considerations, we have not included the systematic uncertainty. This is a limiting factor in our study since one expects a tangible systematic error on the background. The $Z(\rightarrow \nu \bar{\nu})+j$ background can be determined directly from data. One can measure the rate of $Z(\rightarrow \ell \ell)+j$ with the $Z$ decaying into electron or muon pairs. The $Z(\rightarrow \nu \bar{\nu})+j$ cross section can be calculated from the known $Z$ branching ratios. However, the statistical fluctuation will still be too large since $\sum_{\ell=e, \mu} \operatorname{BR}(Z \rightarrow \ell \ell) \approx \sum_{i=e, \mu, \tau} \operatorname{BR}\left(Z \rightarrow \nu_{i} \bar{\nu}_{i}\right) / 3$. Actually it turns out that the $Z(\rightarrow \ell \ell)+j$ sample is by a factor of 5.3 smaller than that of $Z(\rightarrow \nu \bar{\nu})+j$ in the signal region if detector effects are included. The resulting error of the $Z(\rightarrow \nu \bar{\nu})+j$ background is thus $\sqrt{5.3} \approx 2.3$ times larger than the statistical error. Unless the luminosity is very high, the data driven method will not reduce sufficiently the total background uncertainties [46, 47].

In addition, the $W j$ background has a non-negligible systematic error. If one takes the systematic error into account, the signal regions with a harder kinematic cut on the leading jet as well as on the missing transverse momentum perform better than the M1 signal region. In addition, the signal to background ratio improves slightly. However, due to a significant loss of signal events, large integrated luminosities will be required and even then, only small parts of the parameter space will be covered. In practice, we find that the monojet signal is a useful probe of our model if the background is known to within less than one percent. Such a level of precision would be challenging to achieve, yet one should not discount possible developments on the experimental side.

\section{Conclusion}

We have considered the possibility that the Higgs field serves as a portal into a hidden sector endowed with gauge symmetry. Due to the mixing with the hidden "Higgs", the 
$125 \mathrm{GeV}$ scalar observed at the LHC is the only SM particle that couples to the hidden gauge bosons. The latter could either be stable or decay invisibly. If these are sufficiently light, the scenario is already constrained by the Higgs invisible decay.

In this work, we have explored a monojet signature of the Higgsophilic gauge bosons. If these are heavier than about $63 \mathrm{GeV}$ but below half the mass of the heavy "Higgs" $h_{2}$, they can be produced through on-shell decays of $h_{2}$. We find that the statistics allow one to probe invisible decay branching ratios of the heavier Higgs down to $40 \%$, or, in other terms, the hidden sector gauge coupling down to $10^{-1}$. Systematic uncertainties are a limiting factor which must be reduced to within one percent in order to gain the required sensitivity. This also implies that other channels with potentially lower systematic uncertainties, such as vector boson fusion, should be explored in more detail.

\section{Acknowledgments}

This research was supported by the Munich Institute for Astro- and Particle Physics (MIAPP) of the DFG cluster of excellence "Origin and Structure of the Universe". The work of J.S. Kim has been partially supported by the MINECO, Spain, under contract FPA201344773-P; Consolider-Ingenio CPAN CSD2007-00042 and the Spanish MINECO Centro de excelencia Severo Ochoa Program under grant SEV-2012-0249. O.L. acknowledges support from the Academy of Finland project "The Higgs boson and the Cosmos".

Open Access. This article is distributed under the terms of the Creative Commons Attribution License (CC-BY 4.0), which permits any use, distribution and reproduction in any medium, provided the original author(s) and source are credited.

\section{References}

[1] V. Silveira and A. Zee, Scalar phantoms, Phys. Lett. B 161 (1985) 136 [InSPIRE].

[2] R. Schabinger and J.D. Wells, A minimal spontaneously broken hidden sector and its impact on Higgs boson physics at the Large Hadron Collider, Phys. Rev. D 72 (2005) 093007 [hep-ph/0509209] [INSPIRE].

[3] B. Patt and F. Wilczek, Higgs-field portal into hidden sectors, hep-ph/0605188 [INSPIRE].

[4] C. Gross, O. Lebedev and M. Zatta, Higgs-inflaton coupling from reheating and the metastable Universe, arXiv:1506.05106 [INSPIRE].

[5] J. Fan, D. Krohn, P. Langacker and I. Yavin, A Higgsophilic s-channel $Z^{\prime}$ and the CDF $W+2 J$ anomaly, Phys. Rev. D 84 (2011) 105012 [arXiv:1106.1682] [INSPIRE].

[6] S. Gopalakrishna, S. Jung and J.D. Wells, Higgs boson decays to four fermions through an abelian hidden sector, Phys. Rev. D 78 (2008) 055002 [arXiv:0801.3456] [INSPIRE].

[7] O. Lebedev, H.M. Lee and Y. Mambrini, Vector Higgs-portal dark matter and the invisible Higgs, Phys. Lett. B 707 (2012) 570 [arXiv:1111.4482] [INSPIRE].

[8] M. Endo and Y. Takaesu, Heavy WIMP through Higgs portal at the LHC, Phys. Lett. B 743 (2015) 228 [arXiv:1407.6882] [INSPIRE]. 
[9] A. Djouadi, O. Lebedev, Y. Mambrini and J. Quevillon, Implications of LHC searches for Higgs-portal dark matter, Phys. Lett. B 709 (2012) 65 [arXiv:1112.3299] [INSPIRE].

[10] A. Djouadi, A. Falkowski, Y. Mambrini and J. Quevillon, Direct detection of Higgs-portal dark matter at the LHC, Eur. Phys. J. C 73 (2013) 2455 [arXiv:1205.3169].

[11] C.-H. Chen and T. Nomura, Searching for vector dark matter by Higgs portal at the LHC, arXiv: 1507.00886 [INSPIRE].

[12] O. Lebedev and H.M. Lee, Higgs portal inflation, Eur. Phys. J. C 71 (2011) 1821 [arXiv: 1105.2284] [INSPIRE].

[13] P. Langacker, The physics of heavy $Z^{\prime}$ gauge bosons, Rev. Mod. Phys. 81 (2009) 1199 [arXiv:0801.1345] [INSPIRE].

[14] Y. Farzan and A.R. Akbarieh, VDM: a model for Vector Dark Matter, JCAP 10 (2012) 026 [arXiv: 1207.4272] [INSPIRE].

[15] S. Baek, P. Ko, W.-I. Park and E. Senaha, Higgs portal vector dark matter: revisited, JHEP 05 (2013) 036 [arXiv: 1212.2131] [INSPIRE].

[16] C. Gross, O. Lebedev and Y. Mambrini, Non-abelian gauge fields as dark matter, JHEP 08 (2015) 158 [arXiv: 1505.07480] [INSPIRE].

[17] M. Duch, B. Grzadkowski and M. McGarrie, A stable Higgs portal with vector dark matter, JHEP 09 (2015) 162 [arXiv: 1506.08805] [INSPIRE].

[18] G. 't Hooft, A planar diagram theory for strong interactions, Nucl. Phys. B 72 (1974) 461 [INSPIRE].

[19] T. Hambye, Hidden vector dark matter, JHEP 01 (2009) 028 [arXiv:0811.0172] [INSPIRE].

[20] V.V. Khoze, C. McCabe and G. Ro, Higgs vacuum stability from the dark matter portal, JHEP 08 (2014) 026 [arXiv: 1403.4953] [InSPIRE].

[21] A. Falkowski, C. Gross and O. Lebedev, A second Higgs from the Higgs portal, JHEP 05 (2015) 057 [arXiv:1502.01361] [INSPIRE].

[22] N. Kauer and C. O'Brien, Heavy Higgs signal-background interference in $g g \rightarrow V V$ in the Standard Model plus real singlet, Eur. Phys. J. C 75 (2015) 374 [arXiv:1502.04113] [INSPIRE].

[23] V.V. Khoze, G. Ro and M. Spannowsky, Spectroscopy of scalar mediators to dark matter at the LHC and at $100 \mathrm{TeV}$, Phys. Rev. D 92 (2015) 075006 [arXiv: 1505.03019] [InSPIRE].

[24] ATLAS collaboration, Search for new phenomena in final states with an energetic jet and large missing transverse momentum in pp collisions at $\sqrt{s}=8 \mathrm{TeV}$ with the ATLAS detector, Eur. Phys. J. C 75 (2015) 299 [arXiv: 1502.01518] [INSPIRE].

[25] CMS collaboration, Search for invisible decays of Higgs bosons in the vector boson fusion and associated ZH production modes, Eur. Phys. J. C 74 (2014) 2980 [arXiv:1404.1344] [INSPIRE].

[26] ATLAS collaboration, Search for an invisibly decaying Higgs boson produced via vector boson fusion in pp collisions at $\sqrt{s}=8 \mathrm{TeV}$ using the ATLAS detector at the LHC, ATLAS-CONF-2015-004 (2015).

[27] ATLAS collaboration, Search for invisible decays of a Higgs boson produced in association with a $Z$ boson in ATLAS, Phys. Rev. Lett. 112 (2014) 201802 [arXiv:1402.3244] [INSPIRE]. 
[28] ATLAS collaboration, Search for invisible decays of the Higgs boson produced in association with a hadronically decaying vector boson in pp collisions at $\sqrt{s}=8 \mathrm{TeV}$ with the ATLAS detector, Eur. Phys. J. C 75 (2015) 337 [arXiv: 1504.04324] [INSPIRE].

[29] ATLAS collaboration, Search for pair-produced third-generation squarks decaying via charm quarks or in compressed supersymmetric scenarios in pp collisions at $\sqrt{s}=8 \mathrm{TeV}$ with the ATLAS detector, Phys. Rev. D 90 (2014) 052008 [arXiv:1407.0608] [InSPIRE].

[30] P. Nason, A new method for combining NLO QCD with shower Monte Carlo algorithms, JHEP 11 (2004) 040 [hep-ph/0409146] [INSPIRE].

[31] S. Frixione, P. Nason and C. Oleari, Matching NLO QCD computations with Parton Shower simulations: the POWHEG method, JHEP 11 (2007) 070 [arXiv: 0709.2092] [INSPIRE].

[32] S. Alioli, P. Nason, C. Oleari and E. Re, A general framework for implementing NLO calculations in shower Monte Carlo programs: the POWHEG BOX, JHEP 06 (2010) 043 [arXiv: 1002.2581] [INSPIRE].

[33] T. Sjöstrand, S. Mrenna and P.Z. Skands, PYTHIA 6.4 physics and manual, JHEP 05 (2006) 026 [hep-ph/0603175] [INSPIRE].

[34] E. Bagnaschi, G. Degrassi, P. Slavich and A. Vicini, Higgs production via gluon fusion in the POWHEG approach in the SM and in the MSSM, JHEP 02 (2012) 088 [arXiv:1111.2854] [INSPIRE].

[35] P. Nason and C. Oleari, NLO Higgs boson production via vector-boson fusion matched with shower in POWHEG, JHEP 02 (2010) 037 [arXiv:0911.5299] [INSPIRE].

[36] https://twiki.cern.ch/twiki/bin/view/LHCPhysics/CERNYellowReportPageAt14TeV.

[37] T. Gleisberg et al., Event generation with SHERPA 1.1, JHEP 02 (2009) 007 [arXiv:0811.4622] [INSPIRE].

[38] H.-L. Lai et al., New parton distributions for collider physics, Phys. Rev. D 82 (2010) 074024 [arXiv: 1007.2241] [inSPIRE].

[39] S. Frixione, P. Nason and G. Ridolfi, A positive-weight next-to-leading-order Monte Carlo for heavy flavour hadroproduction, JHEP 09 (2007) 126 [arXiv:0707.3088] [INSPIRE].

[40] J. Pumplin, D.R. Stump, J. Huston, H.L. Lai, P.M. Nadolsky and W.K. Tung, New generation of parton distributions with uncertainties from global QCD analysis, JHEP 07 (2002) 012 [hep-ph/0201195] [INSPIRE].

[41] M. Czakon and A. Mitov, Top++: a program for the calculation of the top-pair cross-section at hadron colliders, Comput. Phys. Commun. 185 (2014) 2930 [arXiv:1112.5675] [INSPIRE].

[42] M. Drees, H. Dreiner, D. Schmeier, J. Tattersall and J.S. Kim, CheckMATE: confronting your favourite new physics model with LHC data, Comput. Phys. Commun. 187 (2014) 227 [arXiv: 1312.2591] [INSPIRE].

[43] DELPHES 3 collaboration, J. de Favereau et al., DELPHES 3, a modular framework for fast simulation of a generic collider experiment, JHEP 02 (2014) 057 [arXiv: 1307.6346] [INSPIRE].

[44] J.S. Kim, D. Schmeier, J. Tattersall and K. Rolbiecki, A framework to create customised LHC analyses within CheckMATE, Comput. Phys. Commun. 196 (2015) 535 [arXiv: 1503.01123] [INSPIRE]. 
[45] A.L. Read, Presentation of search results: the CL(s) technique, J. Phys. G 28 (2002) 2693 [INSPIRE].

[46] M. Drees, M. Hanussek and J.S. Kim, Light stop searches at the LHC with monojet events, Phys. Rev. D 86 (2012) 035024 [arXiv:1201.5714] [INSPIRE].

[47] L. Vacavant and I. Hinchliffe, Model independent extra dimension signatures with ATLAS, hep-ex/0005033 [INSPIRE]. 